\section{Numerical investigations into development of seabed trenching in semi-taut moorings}

\section{${ }^{1}$ Chao SUN, Ph.D.}

Geotechnical Engineer, Norwegian Geotechnical Institute, Level 7/40 St Georges Terrace, Perth, WA 6000, Australia.

Formerly, Ph.D. Student, Centre for Offshore Foundation Systems, Oceans Graduate School, University of Western Australia, 35 Stirling Highway, Crawley, Perth, WA 6009, Australia Tel: +61 469743231

Email: chao.sun@ngi.no

\section{${ }^{2}$ Mark Fraser BRANSBY, Ph.D.}

Professor, Centre for Offshore Foundation Systems, Oceans Graduate School, University of Western Australia, 35 Stirling Highway, Crawley, Perth, WA 6009, Australia Email: fraser.bransby@uwa.edu.au

\section{${ }^{3}$ Steven R. NEUBECKER, Ph.D.}

Adjunct Professor, Centre for Offshore Foundation Systems, Oceans Graduate School University of Western Australia, 35 Stirling Highway, Crawley, Perth, WA 6009, Australia Email: steven.neubecker@uwa.edu.au

\section{${ }^{4}$ Mark F. RANDOLPH, Ph.D.}

Professor, Centre for Offshore Foundation Systems, Oceans Graduate School, University of Western Australia, 35 Stirling Highway, Crawley, Perth, WA 6009, Australia Email: mark.randolph@uwa.edu.au

\section{${ }^{5}$ Xiaowei FENG, Ph.D.}

Formerly, Research Fellow, Centre for Offshore Foundation Systems, Oceans Graduate School, University of Western Australia, 35 Stirling Highway, Crawley, Perth, WA 6009, Australia Email: xiaowei.feng@uwa.edu.au

\section{${ }^{6}$ Susan GOURVENEC, Ph.D.}

Professor, School of Engineering, Faculty of Engineering and Physical Sciences,

University of Southampton, Southampton, UK

Email: Susan.Gourvenec@southampton.ac.uk

No. of words (without abstract and references): 5902

No. of tables:

No. of figures: 


\section{Numerical investigations into development of seabed trenching} in semi-taut moorings

\section{ABSTRACT}

Field observations of semi-taut mooring systems have shown severe trench development in front of suction caissons. Trenches extend back from where the chain emerges from the seabed under ambient mooring loads to relatively close to the caisson, and with depths approaching the padeye depth. To help understand the process, a Coupled Eulerian-Lagrangian (CEL) approach has been used to apply cyclic loading to a mooring chain system, treating the chain as a series of linked cylindrical elements and the soil as a strain-softening Tresca material. The chain extended through the soil into the water column, and cyclically changing boundary conditions were applied to the far-end of the chain to reflect a typical mooring layout and metocean conditions. In spite of rather idealized conditions, with loading restricted to the vertical plane of the chain and no consideration of hydrodynamic effects, a stable trench profile developed after only a few cycles of loading. The chain profile straightened during the cycles, with the section nearest to the padeye gradually cutting deeper into the soil and with the amplitude of cyclic motions of the chain, and hence tendency for a trench to form, increasing at shallower depths. The magnitude of soil resistance acting on the chain reduced by increasing cycles, with particularly low resistance in the zone where the trench was most developed. The long-term longitudinal profile of trenching was predicted based on the current modelling, and was found comparable to that inferred from field observations, even without consideration of hydrodynamic and out-of-plane effects.

Keywords: Anchor chain; trenching; coupled Eulerian-Lagrangian; strain-softening; finite element analysis 
The phenomenon of mooring chain trenching - the generation of a 'trench' or void around the chain in the seabed due to operational chain movements - was first discovered during a duediligence inspection of the suction anchors of the Serpentina floating production storage and offloading system (FPSO) offshore Equatorial Guinea (Bhattacharjee et al. 2014). The inspection, conducted after ten years of operation, revealed large trenches that extended from the seabed and almost reached the embedded padeye of the suction anchor (Figure 1(c)). This was considered to threaten the reliability of the anchor system (Alderlieste et al. 2016; Arslan et al. 2015; Sassi et al. 2018) and all anchors were replaced utilizing a larger mooring radius.

Since the first report of significant seabed trenching associated with the mooring chain (Bhattacharjee et al. 2014) there has been widespread industrial interest, as summarized below.

\section{Observations of trenching and trench morphology}

The findings from the Serpentina FPSO inspections (Bhattacharjee et al. 2014) are summarized in Figure 1. Steep sided trenches were observed in front of the suction caissons (Figure 1(a), (b)), with the tensioned chain completely detached from the trench base. Significant motion of the mooring chains was observed in operating swell conditions and identified as the primary cause of trench formation. A schematic of the longitudinal profile of the trenches and the surveyed dimension ranges (Bhattacharjee et al. 2014) are shown in Figure 1(c).

After the Serpentina trenching observations came into the public domain, accurate bathymetry inspections of seabed trenches were conducted for the moorings of five FPSOs and off-loading terminal buoys in the Gulf of Guinea. Colliat et al. (2018) presented longitudinal and transversal trench profiles and statistically related trench development with the mooring-line loads. Initiation of trenching started near the touch-down-point where the most significant chain 
motions occurred, and progressed towards the anchor as a result of chain motions and trench

wall collapses.

Other observations concerning trench evolution were obtained from laboratory floor and centrifuge tests (Sassi et al. 2017). However, fully-developed trenches were not established due to the relatively small time-scale of testing, the small tension amplitudes applied to the chain, and the absence of out-of-plane chain movements or appropriate hydrodynamic modelling.

\section{Investigation of trenching mechanisms and driving factors}

Sassi et al. (2017) described the development of trenching in two stages: formation of the trench with a relatively high trenching rate; and stabilization of the trench with a low trenching growth rate. For the first stage, the trenching rate was linked to the maximum chain tension, with the trenching rate increasing with tension level. During that stage, soil cutting caused by 'flatteningout' of the initial inverse catenary of the embedded chain, together with soil degradation, was proposed as the dominating mechanism. For the second stage, hydrodynamic effects and trench collapse were suggested to explain regressive development of a tunnel and trench back towards the anchor. Accordingly, a time-based 2D analytical tool was developed (Versteele et al. 2017) incorporating chain-soil interaction, soil erosion and trench wall stability.

O'Neill et al. (2018) presented back-calculations of the Serpentina mooring line configurations, from the FPSO fairlead down to the anchor padeye, based on analytical solutions for anchor chains (Neubecker and Randolph 1995), considering various load ranges, different mooring setups and soil properties. Cutting of the chain into soil and lifting up during extreme load conditions were postulated as the primary mechanisms driving the formation of anchor chain trenches. The results showed initial trench formation around the chain near the mudline, although the predicted trench profiles were still far from reaching the limiting geometry of the Serpentina trenches. This was attributed to 'secondary' trench formation mechanisms 
concerning trench collapse and hydrodynamic-related effects not considered in those

calculations.

116

\section{Motivations and scope of present study}

Most of the previous studies of trench development involved direct observations from the field or model tests, providing useful information about the relationship between chain configuration and open trench profile. However, the methods have not addressed chain-soil interaction directly from a geotechnical viewpoint (i.e. soil resistance to the chain, and soil remolding due to chain cutting), although chain-soil interaction is acknowledged as the underlying mechanism for trench development. The analytical tool of (Versteele et al. 2017) predicted trench initiation from the geometry of estimated chain profiles at the extreme load ranges, but their model was phenomenological in nature, without attempting to quantify progressive remolding of soil under repeated loading from the chain.

Consequently, there is still limited knowledge of the trench formation process in the public domain. The numerical work reported in this paper aims to address this gap in knowledge by investigating directly chain-soil interaction during the cyclic loading of a semi-taut mooring system.

The Coupled Eulerian-Lagrangian (CEL) finite-element approach is used here to model the large-deformation problem of a chain cutting cyclically through soil. The approach has been shown (e.g. Kim et al. 2015; Sun et al. 2019a; Zhao and Liu 2016) to capture chain-soil interaction during tensioning of an embedded chain, including incorporation of strain softening to capture soil remolding during chain movement (Sun et al. 2019b). In the analyses presented here, only in-plane cyclic motions of chain in undrained conditions are considered, without consideration of lateral excursions of the mooring line. Hydrodynamic effects, including water entrainment, erosion and scouring of the seabed soil were also not considered in the analyses. 
These simplifications were made in order to allow detailed investigation of the geotechnical

140 The chain system response is investigated by modelling a multi-segment chain which cuts through a softening soil domain cyclically due to the application of a range of practical operational mooring line tensions and angles arising from metocean-induced vessel movements. The behavior of selected chain segments is analyzed to understand how different

144 parts of the embedded chain interact with the seabed during cycling, deforming and remolding

145 the surrounding soil. Distributions of the soil resistance along the chain at different stages of 146 cyclic loading are presented together with implications for the predicted limiting geometry of 147 the trench (within the assumptions made in the numerical analyses). The numerically derived 148 trench is then compared with centrifuge test data for similar soil conditions and evidence from 149 field data, and conclusions are then drawn.

\section{FINITE-ELEMENT MODEL}

\section{Modelling software and approach}

152 All finite element analyses were carried out with the coupled Eulerian-Lagrangian (CEL) 153 approach provided in Abaqus (Dassault Systèmes 2014). The approach used (except for the 154 loading scheme) and parameters selected were as described by Sun et al. (2019a, b). Consequently, only brief details of the methodology are given here.

\section{Chain geometries}

157 The chain was modelled as a string of equally spaced, rigid cylindrical segments, connected 158 using 'LINK' connector elements provided in Abaqus (Figure 2a). Three geometric variables -

159 the length $l_{s}$ and the diameter $d_{s}$ of each segment, and the space between adjacent segments, $s_{s}$

160 - govern the dimensions of the model chain. In the current analyses, $l_{s}$ was taken as $1 \mathrm{~m}$, with $161 s_{s}$ and $d_{s}$ being $0.1 \mathrm{~m}$ and $0.25 \mathrm{~m}$ respectively. 
162

The CEL model was designed to represent a standard $5 d_{b}$ studless chain as shown in Figure $2 a$.

163

164

165

166

167

168

169

170

171

172

173

174

175

176

177

178

179

180

181

182

183

184

185

Each link pair of the $5 d_{b}$ studless chain was idealized as one bar segment together with one spacing, i.e, $l_{s}+s_{s}=6 d_{b}=1.1 \mathrm{~m}$, giving $d_{b} \sim 0.18 \mathrm{~m}$ for this model. This gives $d_{s} / d_{b}=0.25 / 0.18$ $=1.39$ for the configuration adopted, which must be considered when interpreting the soil resistances obtained from the CEL simulations (Sun et al. 2019a).

The global chain model comprised 40 segments (giving a total length of $43.9 \mathrm{~m}$ ) as shown in Figure 2b. The fixed anchor point ('padeye') was set to $9 \mathrm{~m}$ below the soil surface (the anchor depth at Serpentina (Bhattacharjee et al. 2014). Four chain segments, selected for detailed analysis later, are highlighted and numbered \#2, \#13, \#18 and \#25 counting from the padeye. The cyclic motion of the chain was implemented by applying a varying force at appropriate inclination angles at the far end of the chain, as detailed later in this paper.

\section{Soil domain}

The soil was assumed to be fully undrained during chain tensioning and cyclic loading; consequently total stress analyses were performed. The initial (undisturbed) undrained soil strength was assumed to increase linearly with depth $z$, according to $s_{u 0}=2+1.2 z \mathrm{kPa}$, with the effective soil weight $\gamma^{\prime}$ being $2.4 \mathrm{kN} / \mathrm{m}^{3}$. These values correspond with typical properties (specifically the high shear strength ratio $s_{u 0} / \gamma^{\prime} z$, which is expected to be a favorable condition for the stability of trench walls) of seabed sediments offshore West Africa (Ehlers et al. 2005). The effects of parametric variations of soil properties were not investigated herein due to the massive computational expense required for each analysis.

The soil was modelled as a linear elastic-perfectly plastic material, obeying the Tresca yield criterion, but extended here to capture the progressive softening of clay. The undrained shear strength at individual Gauss points was modified immediately prior to re-meshing according to the accumulated absolute plastic shear strain $\xi$, according to (Einav and Randolph 2005): 


$$
S_{u}=\left[\delta_{r e m}+\left(1-\delta_{r e m}\right) \mathrm{e}^{-3 \xi / \xi_{95}}\right] S_{u 0}
$$

187 The models the degradation of soil strength according to an exponential function of cumulative 188 plastic shear strain, $\xi$ ( $\xi_{95}$ representing the value of $\xi$ for $95 \%$ strength degradation), from intact

to fully remolded strength, $\delta_{\text {rem }} S_{u 0}\left(\delta_{\text {rem }}\right.$ being the inverse of sensitivity $\left.S_{t}\right)$. A user subroutine

was implemented in Abaqus to track the evolving soil strength profile. In the present analyses,

$191 \xi_{95}$ and $\delta_{\text {rem }}$ were taken as 10 and 0.2 (giving an equivalent $S_{t}$ of 5, typical of highly structured

192 West African soils).

193 The Young's modulus $E$ was taken as $500 s_{u}$ and Poisson's ratio as 0.49 to approximate

194 incompressible soil response for undrained conditions. The regular mesh comprised 8-noded

195 linear brick elements with reduced integration (EC3D8R in the Abaqus element library). A

196 central fine mesh volume (with a minimum mesh size $L_{e}=0.35 d_{s}$ ) was used in the domain encompassing the predicted chain trajectory during the simulation.

\section{CEL model setup}

199 A global view of the CEL model is presented in Figure 2b together with the detailed chain model illustrated at the bottom of the figure. The total length of the soil domain was taken as $57.7 \mathrm{~m}$, and the out-of-plane thickness as $30 \mathrm{~d}_{\mathrm{s}}(7.5 \mathrm{~m})$ to avoid boundary effects, with the line of symmetry along the vertical axial of the chain used to halve the domain size. A layer of 'void' with height $2 l_{s}(2 \mathrm{~m})$ was prescribed above the soil surface to accommodate possible upward soil movement during the periodic tensioning process.

The anchor chain was assumed to be weightless and initially 'wished-in-place' in the postinstallation configuration shown in Figure 2b. Ignoring the chain weight has been shown to make negligible difference to the chain response, especially given the strength intercept of

$2 \mathrm{kPa}$ at the soil surface (Sun et al., 2019a, b). A vertical section was fully embedded and attached to the padeye, and a horizontal section was laid on the seabed (with the centerline level at the mudline). The chain had boundary conditions of full displacement (but not rotational) 
fixity at the padeye (leading to tension $T_{a}$ applied at an angle $\theta_{a}$ to the horizontal at the padeye)

reacting to tension $T_{m}$ applied at an angle $\theta_{m}$ at the 'dragging point'. Note that the chain tension

and angle do not change between mudline intersection and dragging point since the chain is

214 weightless.

215 The chain was first loaded horizontally at the seabed assuming a slack catenary of the mooring

216 line during pre-tensioning (increasing $T_{m}$ gradually maintaining $\theta_{m}$ zero), and then tensioned

217 with periodically increasing and decreasing mudline loads and load inclinations to the

218 horizontal. The relationship between the mudline tension and inclination angle (i.e. $T_{m}-\theta_{m}$ 219 relationship) was selected to model the global behavior of a practical mooring setup as 220 discussed below.

\section{$221 \quad$ Loading scheme based on a practical mooring setup}

222 The boundary conditions for loading the chain (i.e. applied $T_{m}$ and $\theta_{m}$ for the chain) in the CEL model were chosen to reflect a real mooring configuration, decoupling the mooring line section through the water column from the embedded chain section and assuming a fixed touch-down point on the seabed, consistent with engineering design practice. The mooring configuration was designed to recreate approximately the Serpentina mooring (Bhattacharjee et al. 2014; O'Neill et al. 2018) shown in Figure 3a. The length of mooring line in the water was set as $1000 \mathrm{~m}$ from the fixed touch-down point (with tension $T_{m}$ and angle $\theta_{m}$ ), up to the fairlead (with tension $T_{s}$ and angle $\theta_{s}$ ) connecting to the floating facility with a corresponding water depth of

$230475 \mathrm{~m}$ (averaging the depths reported for Serpentina). As illustrated in Figure 3a four sections make up the whole $1000 \mathrm{~m}$ line with different buoyant weights $w_{\text {mooring, }}$ sourced from Vryhof

232 (2015) according to the types of chain and wire rope used in the Serpentina mooring system 233 (Bhattacharjee et al. 2014). 
With the assumption of constant water depth and length of the mooring line suspended in water,

the relationship between $T_{m}$ and $\theta_{m}$ for increasing fairlead movements (i.e. increasing $\mathrm{T}_{\mathrm{s}}$ ) was deduced by integrating the equilibrium equations of the mooring line (see Figure 3a) over the whole length from the touch-down point to the fairlead, following Faltinsen (1993). The derived mudline tension $T_{m}$ ranges from $640 \mathrm{kN}$ (with mudline angle $\theta_{m}=0^{\circ}$ ) to $2860 \mathrm{kN}$ (with angle $\theta_{m}=22^{\circ}$ ) with the relationship shown on Figure 4. This range of mudline tension falls within the design range (500 kN for pre-tensioning, and a maximum value of $3000 \mathrm{kN}$ considering the extreme conditions) adopted by O'Neill et al. (2018) based on the Serpentina moorings.

The derived configurations of the mooring line are plotted on the left of Figure 3b together with the changing tension and angle at two ends of the line. A mudline chain angle, $\theta_{m}$ of $0^{\circ}$ with $T_{m}$ $=640 \mathrm{kN}$ was designated as the completion of the pre-tensioning stage, while $\theta_{m}=10^{\circ}\left(T_{m}=\right.$ $980 \mathrm{kN})$ and $\theta_{m}=22^{\circ}\left(T_{m}=2860 \mathrm{kN}\right)$ were selected as the lower and upper limits respectively for cyclic loading of the mooring line. The lower limit $\left(\theta_{m}\right.$ of $10^{\circ}$ and $T_{m}$ of $\left.980 \mathrm{kN}\right)$ during cycling was selected to correspond to the normal operational condition of the mooring line. The lateral movement (drift) of the floating facility during cyclic loading condition is indicated on the right of Figure 3b. The maximum load is generated by $17 \mathrm{~m}$ of drift from the virtual completion of pre-tensioning and $7 \mathrm{~m}$ of movement from the operational $\left(\theta_{\mathrm{m}}=10^{\circ}\right)$ condition. This displacement range is comparable to the analyses of O'Neill et al. (2018) (26 m drift due to a larger tension range of $500 \leq T_{m} \leq 3000 \mathrm{kN}$ ).

Figure 4 shows the non-linear mudline tension - mudline chain angle relationship obtained from the above in-water chain analyses. The relationship compares reasonably with the analytical solution by O'Neill et al. (2018), who considered a constant length of mooring line (942 m) with depths of seawater and padeye being $436 \mathrm{~m}$ and $9 \mathrm{~m}$, respectively.

Using the relationship in Figure 4, the loading sequence in the CEL modelling consisted of the following stages: 
1. During pre-tensioning the mudline tension $T_{m}$ was increased linearly with time from 0 to $640 \mathrm{kN}$ while $\theta_{m}$ remained zero.

2. For the first loading step (labelled 'Load \#1'), both $T_{m}$ and $\theta_{m}$ were increased from $640 \mathrm{kN}$ to $2860 \mathrm{kN}\left(\theta_{m}\right.$ increasing from $0^{\circ}$ to $\left.22^{\circ}\right)$.

3. The load was reduced (in stage 'Unload \#1') until $T_{m}=980 \mathrm{kN}\left(\theta_{m}=10^{\circ}\right)$.

4. Repeated loading (Load \#2, \#3 etc.) and unloading (Unload \#2, \#3 etc.) were implemented alternately between the same limits of mudline tension and mudline angle as for cycle 1.

Seven cycles of loading between the lower and upper limits were completed. The tremendous required on a fast server to run these seven cycles.

\section{RESULTS}

\section{Chain system response}

272 Figure 5a presents the mudline and padeye tensions $T_{m}$ and $T_{a}$ against simulation time from the 273 initial pre-tensioning phase through the eight loading and seven unloading cycles. During pre274 tensioning, the chain tension at the anchor padeye, $T_{a}$, is only slightly smaller than the mudline tension $T_{m}$. The small difference $\left(T_{a} / T_{m} \approx 0.97\right)$ reflects the low mobilization of frictional chain resistance because of the combined effects of high mobilization of normal resistance over most of the chain (Sun et al. 2019a) and the greater reduction in frictional resistance than normal resistance during remolding (Sun et al. 2019b). For the subsequent cyclic loading, $T_{a}$ is within $1 \%$ of $T_{m}$.

Figure $5 \mathrm{~b}$ plots the corresponding changes of the mudline and padeye angles $\theta_{m}$ and $\theta_{a}$ with

281 simulation time. The variation of the chain angle at the mudline, $\theta_{m}$, is a saw-toothed response 282 as specified by the load scheme. In contrast, the chain angle at the padeye $\theta_{a}$ decreased 
with a gradually decreasing rate, as the chain cut through the soil to the fully loaded geometry.

It then reduced further in shallow steps with marginal decreases in chain angle at the end of each loading phase. The padeye angle $\theta_{a}$ decreased continuously, becoming smaller than the mudline $\theta_{m}$ on application of Load \#6 and subsequent cycles of maximum load application.

Figure 6 shows the configurations of the chain at the completion of selected loading and unloading stages with dots representing the positions of the chain link elements. In the figure, black represents the end of pre-tensioning ( $\mathrm{P}$ on Figure 5), red shows the end of loading $\left(\mathrm{L}_{1}\right)$ and unloading $\left(\mathrm{U}_{1}\right)$ stages of the first cycle, and blue shows the equivalent stages for the seventh cycle. For the stages where much of the chain was moving downwards and so penetrating deeper into the soil, e.g. during pre-tensioning and the subsequent unloading stages, the chain became less curved with cycle number (i.e. having less of an inverse catenary shape) with the difference of the chain angles at the padeye and mudline becoming smaller. During each loadup cycle, the chain straightened, apart from the deeply embedded section very close to the padeye, with the chain becoming straighter in each subsequent cycle so that by loading \#7, $\theta_{m}$ $\approx \theta_{a}$ (as already pointed out on Figure $5 \mathrm{~b}$ ). Overall, as cycling proceeded, the chain penetrated deeper into the seabed (e.g. comparing the difference between the blue and the red profiles in Figure 6).

\section{Observation of the numerical trenching phenomenon}

The numerically-generated trench is shown in Figure 7 for five different stages, $P, L_{1}, U_{1}, U_{7}$ and $\mathrm{L}_{8}$ (as annotated in Figure $5 \mathrm{a}$ ). The grey regions indicate that the Eulerian domain is completely filled with soil material, and the black regions indicate gap formation. Further insight is provided in Figure 8, which shows cross-sections normal to the plane of the chain for two positions along the chain (with the chain indicated as a blue half circle). The elevations in 
opening below the chain, with partial collapse of the lifted soil at the unload points. With

increasing cycles the trench becomes more developed, with the chain near the mudline (beyond about segment \#15) essentially moving within an open trench, while a smaller tunnel develops in the mid-region (difficult to see in Figure 7, but extending from about segment \#13 to \#15). The cross-sectional views (Figure 8(a) and (b)) at the centroids of segments (\#18 and \#23) confirm the trench opening near the mudline during the initial chain lifting (stage $\mathrm{L}_{1}$ ) and then

314 the development of the trench (in stages $L_{1}$ to $L_{8}$ ). The trench develops as the chain moves up and down between loading and unloading stages whilst progressively penetrating the trench bottom downwards during unloading.

The limitations of the CEL analyses, without consideration of hydrodynamic effects, water entrainment and out-of-plane motions, do not allow full trench development, or the observed breadth of the open trenches (reaching 50\% of the trench depth) reported by Colliat et al. (2018). Instead, cells partially filled with remolded soil remain above the chain (see Figure 8).

321 However, the main processes and consequences of trench development are clear, even within the quite limited number of loading cycles that were feasible computationally.

\section{Response of chain at selected segments}

\section{Overall displacements}

325 To understand how different parts of the chain interact with the seabed, the accumulation of 326 two displacement components (locally normal and axial to the chain axis) are plotted for four 327 selected chain segments in Figure 9, with the segment locations indicated in Figure 6 and Figure 328 7. The graphs start from the 'wished-in-place' installed configuration (i.e. the beginning of the 329 simulation) until the completion of Load \#4 (L4).

330 The accumulated displacement graphs show the domination of the normal movement for segment \#2 (near the padeye) due to the constraint at the padeye (which precludes any axial chain movement, but allows chain rotation), while axial movements dominate normal 
movements closer to the dragging point (e.g. for segment \#25). The accumulated axial

displacement of the four segments remained almost constant after $\mathrm{L}_{1}$, irrespective of the axial movements during pre-tensioning, reflecting a relatively taut configuration of the chain during cycling.

\section{Normal displacements}

In contrast to the axial displacements, the normal displacements of the four segments show distinctive responses during cycling after the initial increase of normal displacement, with the chain cutting through the soil, during pre-tensioning. The tension-displacement responses during all seven loading cycles are plotted in detail in Figure 10, with the loading stage of each curve presented in black and the unloading stages in magenta.

The normal movement of segment \#2 (near the padeye) increases 'step-wise' with reducing step heights with increasing numbers of cycles (Figure 10a). No significant movement occurs during unloading stages, while normal displacement increases mainly during the later stage of each loading, reducing progressively with cycle number.

The normal displacement of segment \#13 (about half way between the padeye and the seabed surface) increases (cutting further downwards into the soil) during the first half of each loading stage, then decreases (being lifted up) over the second half of the loading stage before increasing further during unloading of the chain (see Figure 10b). Interestingly the cyclic range of normal displacement increases with cycle number even though there is a reduction in the cumulative cycle-by-cycle chain segment movement. This reflects the development of a gap or tunnel around that part of the chain.

Segments \#18 and \#25 (see Figure 10c and d), which are near the seabed surface, show more conventional cyclic motion with alternate directions of movement during unloading and loading with the cyclic amplitude not changing very much. In this zone, the very low soil resistance, 
much greater displacement amplitudes than for the deeper chain segments.

\section{Normal resistances}

The relationships between normal resistance and displacement for the selected segments are presented in Figure 11, with black lines represent loading stages and magenta unloading. Normal soil resistances are shown normalized by the ultimate normal seabed resistance, $Q_{u l t}$, for non-softening soil at the instantaneous chain depth.

The ultimate resistance $Q_{u l t}\left(=N_{b, u l t} d_{b} S_{u 0}=19 d_{b} S_{u 0}\right)$ was derived based on monotonic penetration analyses for a deeply embedded multi-segment chain section in non-softening soil (Sun et al. 2019a), benchmarked by Degenkamp and Dutta (1989). For simplicity, the ‘deep’ ultimate resistance was adopted for the normalization even for the shallow part of the chain. In softening soil with sensitivity $S_{t}=5$, the post-peak steady-state normal resistance (for monotonic chain movement in deep conditions) was $0.68 Q$ ult (Sun et al. 2019b) and this will theoretically reduce to $0.2 Q_{\text {ult }}$ for fully remolded conditions. Lower resistance will be obtained near the seabed surface or as gapping develops.

In current design methods (Degenkamp and Dutta 1989; Neubecker and Randolph 1995), the soil resistance to the chain is conventionally decomposed as two independent components, the normal resistance $Q$ and 'frictional' resistance $F$, which are expected to react in opposition to the normal and axial incremental displacements. Consequently, positive normal resistance is defined as that corresponding to downward movement of the chain (as would occur during the initial tensioning), and negative resistance the result from upward movement. The frictional resistance during chain cycling remained close to zero as the axial movement became insignificant after Load \#1 (see Figure 9) and is not considered further.

The behavior of segment \#2 (Figure 11a) indicates that this section of chain gradually penetrates deeper into the seabed with increasing cycles and that the normal seabed resistance gradually 
during which there is downward plastic penetration of the chain into less disturbed soil, with

slight upward movement during chain unloading that lead to sharp drops in normal resistance.

During the small upward movements there appears to be no detachment of the chain from the underlying soil, since the net resistance remains positive. Although the cumulative chain movement is downwards, the soil resistance reduces gradually with increasing cycle number from approximately $Q / Q_{u l t}=0.6$ at $L_{1}$ (which is broadly consistent with the 0.68 expected for the first movement for $S_{t}=5$ soil) to $Q / Q_{u l t} \approx 0.35 \mathrm{for} L 7$, which reflects increasing remolding of soil around the chain. Further cycles are expected to continue to reduce $Q / Q_{u l t}$ during the loading phases as the soil continues to remold.

Segments \#13, \#18 and \#25 experience two-way oscillation of normal resistance, reaching maximum positive resistance (against downward movement) partway through each loading cycle as reflected in the changes in normal displacement direction shown on Figure 11. The maximum uplift resistance is reached towards (or at) the end of the chain loading (i.e. at $\mathrm{L}_{1}, \mathrm{~L}_{2}$ etc.). For all three segments the cyclic range of normalized resistance decreases continuously, reflecting a decrease in penetration resistance from an initial value of $Q / Q_{u l t} \approx 0.68$ down towards $Q / Q_{\text {ult }} \approx 0.4$, while the uplift resistance decreases in magnitude from $Q / Q_{\text {ult }} \approx-0.4$ down to -0.32 to -0.35 for segments \#13 and \#18. For segment \#25, a maximum uplift resistance was $Q / Q_{u l t} \approx-0.1$ partway through the loading stage, but reduced towards zero by the end of loading. These responses reflect the combined effects of soil remolding for the deeper conditions, and gapping and trench development during uplift, particularly for segment \#25. The chain response in the vicinity of segment \#25 appears similar to that of a vertically oscillating steel catenary riser in the touchdown (Bridge et al. 2004; Randolph and Quiggin 2009). This part of the chain lifts out of the soil during each cycle, experiencing a small amount of uplift resistance $\left(-0.1<Q / Q_{u l t}<0.1\right)$ until the chain fully loses contact with the seabed (when 
$Q \approx 0$ ). During reduction in tension, the chain segments repenetrate the seabed, pushing initially

into remolded soil before coming into contact with 'new' soil. In each cycle, the chain

penetrates slightly further into the seabed at the end of unloading with penetration resistance decreasing from $0.6 Q_{u l t}$ to $0.5 Q_{u l t}$ with each cycle (and still reducing at $\mathrm{U}_{7}$ ).

\section{Soil resistance profiles}

412 Figure 12 presents depth profiles of normal soil resistance at the completion of the loading 413 (plotted on the left) and unloading (plotted on the right) phases of each cycle.

414 The normal resistance curves at the completion of the loading phases show three types of chainsoil interaction:

(i) For the deeply embedded segments near the padeye (where the chain moves downwards during system tensioning), positive seabed resistance is mobilized. This reduced with number of cycles because of remolding. Segment \#2 shows this behavior.

(ii) Further away from the padeye, the soil resistance is negative because the chain moves upwards at the end of tensioning. The resistance stays approximately constant with depth due to the deep uplift mechanism, although for any given segment the resistance gradually reduced with cycle number. Segment \#13 shows this type of behavior, which appears to correspond to the 'tunneling' mechanism identified earlier in Figure 7.

(iii) For chain segments near the mudline the absolute value of the negative (uplift) soil resistance during chain loading gradually reduces towards zero due to mobilization of shallow uplift resistance mechanisms and gradual trench development. Slight uplift resistance was generated even for some segments above the mudline because 
As cycling proceeded, the depth range of the positive (penetration) resistance section reduced,

i.e. the transition to cyclic penetration and uplift resistance moves deeper, with the maximum normal resistance in this section reducing from $0.6 Q_{\text {ult }}$ to just below $0.4 Q_{u l t}$. The seabed resistance reverses (resisting uplift) during Load \#2 for segment \#11, Load \#4 for segment \#9 and Load \#6 for segment \#8, reflecting the transition point moving gradually closer to the padeye with increasing number of cycles. The changing depth of this transition point with numbers of cycles is shown on the left side of Figure 12, with the depth ranges of each observed mechanism identified.

In contrast, the normal resistance profiles at the completion of each unloading phase shown on the right of Figure 12 show that resistance remains positive for the whole chain length because the whole chain is moving downwards at the end of each unloading phase (as can be seen in Figure 10). Within the first two unloading events, the magnitude of the soil resistance near the padeye reduces to just above $0.1 Q_{u l t}$ with the near-mudline resistance remaining between 0.5 and 0.6 of the ultimate intact resistance (apart from a zone within the top $0.5 \mathrm{~m}$ of the seabed where resistance reduced to almost zero on unloading). With increasing cycles, the resistance at all depths reduces, although this happens increasingly slowly as the seabed surface is approached because the chain moves progressively into 'new' soil as it works its way down into the seabed. It is postulated that, if sufficient cycles were conducted, the ultimate condition of the normal resistance during chain unloading might be for none of the segments below the mudline to mobilize more than about $0.1 Q_{\text {ult }}$ considering the near-surface and gapping effects for soil in different embedment depths.

\section{IMPLICATIONS FOR TRENCHING}

Figure 13 brings together the findings about loadings and displacements on individual chain segments and observations about seabed geometry changes (particularly trench and gap 
(a) a 'trenching' zone (from the seabed to about $3 \mathrm{~m}$ ) where a shallow uplift mechanism prevails, the resistance on the chain reverses direction during system loading and unloading and a trench is generated with soil at the seabed surface elevated. The extent of this zone appears to be governed by the chain oscillating upwards and downwards during system loading (as hypothesized by O'Neill et al. (2018)) and where shallow chain uplift mechanisms (with gap formation beneath the chain) occur (the value of $z / d_{s}$ where this occurs may be estimated knowing $\gamma^{\prime} d_{s} / s_{u}$, e.g. Merifield et al. (2009)).

(b) a 'tunneling' zone (from about $3 \mathrm{~m}$ to $6.5 \mathrm{~m}$ for $\mathrm{L}_{8}$ ) where the soil resistance reverses during loading and unloading, but the local uplift mechanism is local rather than reaching the mudline. A small 'tunnel' is generated above and below the chain due to detachment behind the chain during movement (see Figure 13). The depth of the tunneling zone increases with number of cycles (see Figure 12).

(c) a 'remolding' zone (from the padeye to $6.5 \mathrm{~m}$ for $\mathrm{L}_{8}$ ) where the soil resistance remains below the chain although small chain movements gradually remold the soil around the chain and the resistance reduces with numbers of cycles. Gapping was not observed in this zone.

If water entrainment and hydrodynamic effects (with scour) are included, the soil around the 'shallow uplift' section of the chain is likely to be transported away with time. Furthermore, the soil opening in the 'tunneling' section provides an additional water ingress path, which is likely to lead to scour and softening of this zone and so this zone is also expected to trench with time. The lowest depth of the tunneling zone in the CEL analysis was observed to increase during cycling with no equilibrium position achieved in the eight cycles modelled. The rate of tunnel development is likely to accelerate in reality as soil is removed though hydrodynamic effects, 

progressively down towards the padeye.

The trenching process discussed above is compared with the field observations from Bhattacharjee et al. (2014) and with the centrifuge observations from Sassi et al. (2017). The lowest extent of the 'tunnel' zone in the CEL analyses (for only eight cycles) approaches the depth of the trench in the field observation. Furthermore, the end of centrifuge test geometry (with 1000, small amplitude cycles) appears qualitatively similar to the numerical result. Both provide confidence in the simulation method despite the simplifications made.

With the help of the analytical solutions of the embedded chain by Neubecker and Randolph (1995), a predicted limiting configuration of chain at minimum tension was derived with the assumption that the normal soil resistance was reduced to $0.1 Q_{\text {ult }}$ with no friction mobilized based on the previous discussion with reference to Figure 11 and Figure 12. This is shown on Figure 13 and is compared with the inferred longitudinal profile of trenches by Bhattacharjee et al. (2014). The difference can be attributed to ‘secondary' trenching mechanisms including hydrodynamic transportation (O'Neill et al. 2018). The analytical prediction for the limiting trench geometry based on CEL modelling results shows an improvement compared with the previous results by O'Neill et al. (2018) even without consideration of hydrodynamic effects.

\section{CONCLUSIONS}

Abaqus CEL was utilized to analyze the behavior of a mooring chain embedded in the seabed during cyclic loading of a semi-taut mooring system. The analysis allowed identification of patterns of cyclic chain movements and chain-soil interaction and provided a quantitative basis for understanding how chain trenching develops.

The embedded section of the chain demonstrated three different types of mechanisms, which were described as 'trenching', 'tunneling' and 'remolding'. In the 'trenching' section the chain 
lifted the soil above the chain, opening up a 'slot' in the seabed with the chain penetrating slightly further into the seabed during each unloading stage. The adjacent 'tunneling' section of the chain opened a smaller gap above and below the chain. Both zones were associated with seabed uplift resistance mechanisms during tensioning of the chain.

Once hydrodynamic effects and out of plane motion of the chain are considered (neither of which were modelled in the analysis), the combined 'trenching' and 'tunneling' zones are likely to define the final extent of chain trenching. The lower extent of the tunneling zone moved closer to the padeye with number of cycles, suggesting that some degree of trenching will progress down the chain to the padeye during operation. Water entrainment will also contribute to growth of the trench.

Finally, all chain segments were observed to move down into the soil during chain unloading, remolding the soil and reducing the seabed penetration resistance and leading ultimately to an almost straight chain (i.e. with minimal soil resistance) throughout loading cycles. The chain profile during unloading, assuming fully remolded soil resistance, approximates the extent of the trenching zone and appears to agree closely with observations by (Bhattacharjee et al. (2014)).

Despite the limitations of the 2D in-plane motion of the chain analyzed, the small number of cycles (limited by computational expense) and the lack of consideration of hydrodynamic effects and other aspects such as out of plane motion and water entrainment, all of which would lead to further growth of the trench, the CEL analysis led to significant improvement in understanding the chain trenching process.

\section{DATA AVAILABILITY STATEMENT}

All data, models, or code that support the findings of this study are available from the corresponding author upon reasonable request. 
This work forms part of the activities of the Centre for Offshore Foundation Systems.

531 Established in 1997 under the Australian Research Council’s Special Research Centres Program and currently supported as a node of the Australian Research Council’s Centre of Excellence for Geotechnical Science and Engineering (ARC grant CE110001009), and through the Fugro Chair in Geotechnics, the Lloyd's Register Foundation Chair and Centre of Excellence in

535 Offshore Foundations and the Shell EMI Chair in Offshore Engineering. The first author is 536 supported with an Australian Government Research Training Program Scholarship and a Fugro 537 PhD Scholarship, the second author is supported as the Fugro Chair in Geotechnics and the fifth 538 author is supported by the National Natural Science Foundation of China (No. 51890915). All 539 the support above is gratefully acknowledged.

\section{REFERENCES}

541 Alderlieste, E. A., Romp, R. H., Kay, S., and Lofterød, A. (2016). "Assessment of Seafloor

542 Trench for Suction Pile Moorings: a Field Case." In Proc., Offshore Technology Conference, 543 Offshore Technology Conference.

544 Arslan, H., Peterman, B. R., Wong, P. C., and Bhattacharjee, S. (2015). "Remaining Capacity 545 of the Suction Pile due to Seabed Trenching." In Proc., The Twenty-fifth International 546 Offshore and Polar Engineering Conference, International Society of Offshore and Polar 547 Engineers.

548 Bhattacharjee, S., Majhi, S. M., Smith, D., and Garrity, R. (2014). "Serpentina FPSO Mooring 549 Integrity Issues and System Replacement: Unique Fast Track Approach." In Proc., Offshore 550 Technology Conference, Offshore Technology Conference.

551 Bridge, C., Laver, K., Clukey, E., and Evans, T. (2004). "Steel catenary riser touchdown point 552 vertical interaction models." In Proc., Offshore Technology Conference, Offshore Technology 553 Conference.

554 Colliat, J.-L., Safinus, S., Boylan, N., and Schroeder, K. (2018). "Formation and Development 555 of Seabed Trenching from Subsea Inspection Data of Deepwater Gulf of Guinea Moorings." 556 In Proc., Offshore Technology Conference, Offshore Technology Conference. 
Dassault Systèmes (2014). ABAQUS 6.14 Analysis User's Manual, Simulia Corp, Providence,

558

559

560

561

562

563

564

565

566

567

568

569

570

571

572

573

574

575

576

577

578

579

580

581

582

583

584

585

586

587

588

589 RI, USA.

Degenkamp, G., and Dutta, A. (1989). "Soil resistances to embedded anchor chain in soft clay." Journal of Geotechnical Engineering, 115(10), 1420-1438.

Ehlers, C. J., Chen, J., Roberts, H. H., and Lee, Y. C. (2005). "The origin of near-seafloor crust zones in deepwater." In Proc., Proc. Int. Symp. on Frontiers in Offshore Geotechnics: ISFOG, 927-934.

Einav, I., and Randolph, M. F. (2005). "Combining upper bound and strain path methods for evaluating penetration resistance." International Journal for Numerical Methods in Engineering, 63(14), 1991-2016.

Faltinsen, O. (1993). Sea loads on ships and offshore structures, Cambridge university press.

Kim, Y., Hossain, M. S., and Wang, D. (2015). "Dynamically Installed Anchors: Performance of embedded mooring chain profile in clay." Frontiers in Offshore Geotechnics III, Meyer, ed., Taylor \& Francis Group, London, 881-886.

Merifield, R. S., White, D. J., and Randolph, M. F. (2009). "Effect of surface heave on response of partially embedded pipelines on clay." Journal of Geotechnical and Geoenvironmental Engineering, 135(6), 819-829.

Neubecker, S. R., and Randolph, M. F. (1995). "Profile and frictional capacity of embedded anchor chains." Journal of geotechnical engineering, 121(11), 797-803.

O'Neill, M., Erbrich, C., and McNamara, A. (2018). "Prediction of Seabed Trench Formation Induced by Anchor Chain Motions." In Proc., Offshore Technology Conference, Offshore Technology Conference.

Randolph, M., and Quiggin, P. (2009). "Non-linear hysteretic seabed model for catenary pipeline contact." In Proc., ASME 2009 28th International Conference on Ocean, Offshore and Arctic Engineering, American Society of Mechanical Engineers, 145-154.

Sassi, K., Kuo, M. Y. H., Versteele, H., Cathie, D. N., and Zehzouh, S. (2017). "Insights Into the Mechanisms of Anchor Chain Trench Formation." In Proc., Offshore Site Investigations and Geotechnics Conference, Society for Underwater Technology, SUT OSIG, London, UK, 10-12.

Sassi, K., Zehzouh, S., Blanc, M., Thorel, L., Cathie, D., Puech, A., and Colliat-Dangus, J.-L. (2018). "Effect of Seabed Trenching on the Holding Capacity of Suction Anchors in Soft Deepwater Gulf of Guinea Clays." In Proc., Offshore Technology Conference, Offshore Technology Conference. 
590 Sun, C., Feng, X., Bransby, M. F., Neubecker, S. R., Randolph, M. F., and Gourvenec, S.

591 (2019b). "Numerical investigations of the effect of strain softening on the resistance to

592 embedded mooring chains." Applied Ocean Research, 92, 101944.

593 Sun, C., Feng, X., Neubecker, S. R., Randolph, M. F., Bransby, M. F., and Gourvenec, S.

594 (2019a). "Numerical study of mobilized friction along embedded catenary mooring chains."

595 Journal of Geotechnical and Geoenvironmental Engineering 145(10), 04019081.

596 Versteele, H., Kuo, M. Y. H., Cathie, D. N., Sassi, K., and Zehzouh, S. (2017). "Anchor

597 Chain Trenching-Numerical Simulation of Progressive Erosion." In Proc., Offshore Site

598 Investigations and Geotechnics Conference, Society for Underwater Technology, SUT OSIG,

599 London, UK, 10-12.

600 Vryhof (2015). "Anchor Manual 2015." Global Maritime Vryhof Anchors.

601 Zhao, Y., and Liu, H. (2016). "Numerical implementation of the installation/mooring line and

602 application to analyzing comprehensive anchor behaviors." Applied Ocean Research, 54, 101603114.

604 


\section{Figure captions}

606 Figure 1. Surveys and schematic of the seabed trench at Serpentina FPSO (Bhattacharjee et al., 2014): (a) ROV images of seabed trenching in the vicinity of the suction caisson; (b) Sonar showing proximity of trenches to suction caissons; (c) Schematic of the trench with

609 surveyed dimensions.

610 Figure 2. CEL modelling of the embedded chain: (a) Chain model representing $5 d_{b}$ studless 611 chain; (b) FE model setup.

612 Figure 3. Setup of the mooring line for CEL modelling work: (a) A practical example of a 613 mooring line in the seawater; (b) Configurations of the mooring line and the corresponding 614 boundaries of the tension and angle.

615 Figure 4. Load input from the mooring line in the seawater.

616 Figure 5. Tensions and angles of the chain during the complete simulation: (a) Mudline and 617 padeye tension; (b) Mudline and padeye angles.

618 Figure 6. Comparison of the configurations of the embedded chain at selected instants during 619 cyclic loading.

620 Figure 7. Volume fraction of soil at selected instants during cyclic loading.

621 Figure 8. Cross-sectional views of the volume fraction of soil at selected instants during 622 cyclic loading.

623 Figure 9. Accumulated displacement of selected chain segments (from pre-tensioning to Load 624 \#4): (a) Segment \#2; (b) Segment \#13; (c) Segment \#18; (d) Segment \#25.

625 Figure 10. Variation of normal displacement during cyclic loading for selected chain segments 626 (a) Segment \#2; (b) Segment \#13; (c) Segment \#18; (d) Segment \#25.

627 Figure 11. Normal resistance-displacement response for selected chain segments: (a) Segment 628 \#2; (b) Segment \#13; (c) Segment \#18; (d) Segment \#25.

629 Figure 12. Evolution of the depth profile of normal soil resistance to the chain at selected 630 instants during cyclic loading.

631 Figure 13. Implications about the development of trenching within the scope of current 632 model: (a) CEL modelling; (b) Centrifuge model test. 


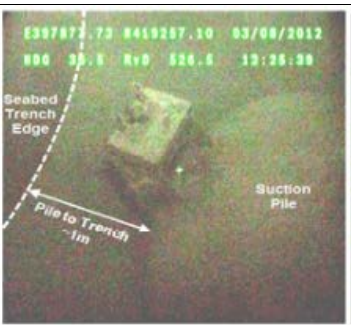

(a)

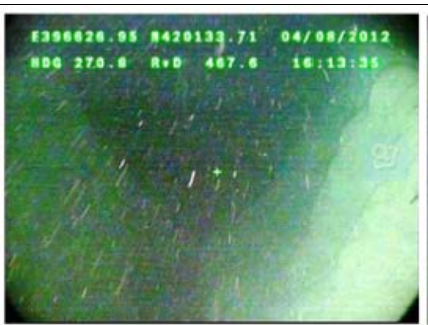

Opening of the Trench

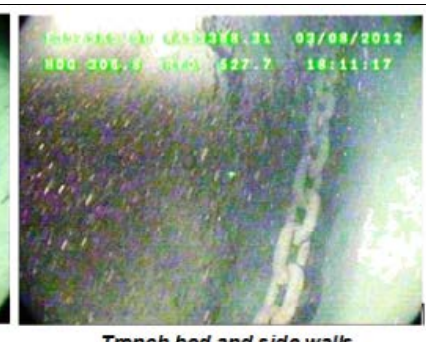

Trench bed and side walls

(b)
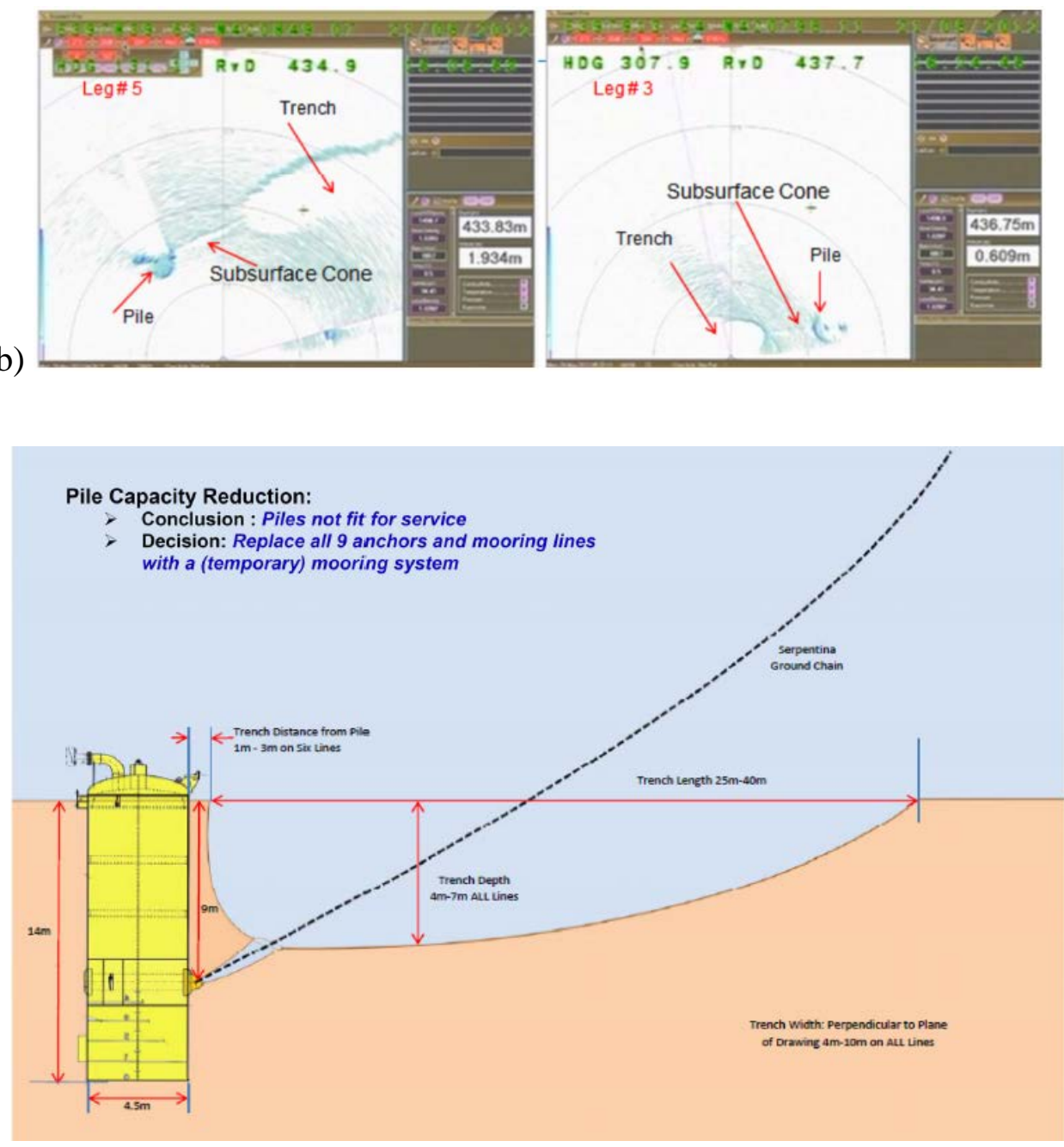
(c)

637 Figure 1. Surveys and schematic of the seabed trench at Serpentina FPSO (Bhattacharjee et al., 2014): (a) ROV images of seabed trenching in the vicinity of the suction caisson; (b)

639 Sonar showing proximity of trenches to suction caissons; (c) Schematic of the trench with surveyed dimensions. 
(a)
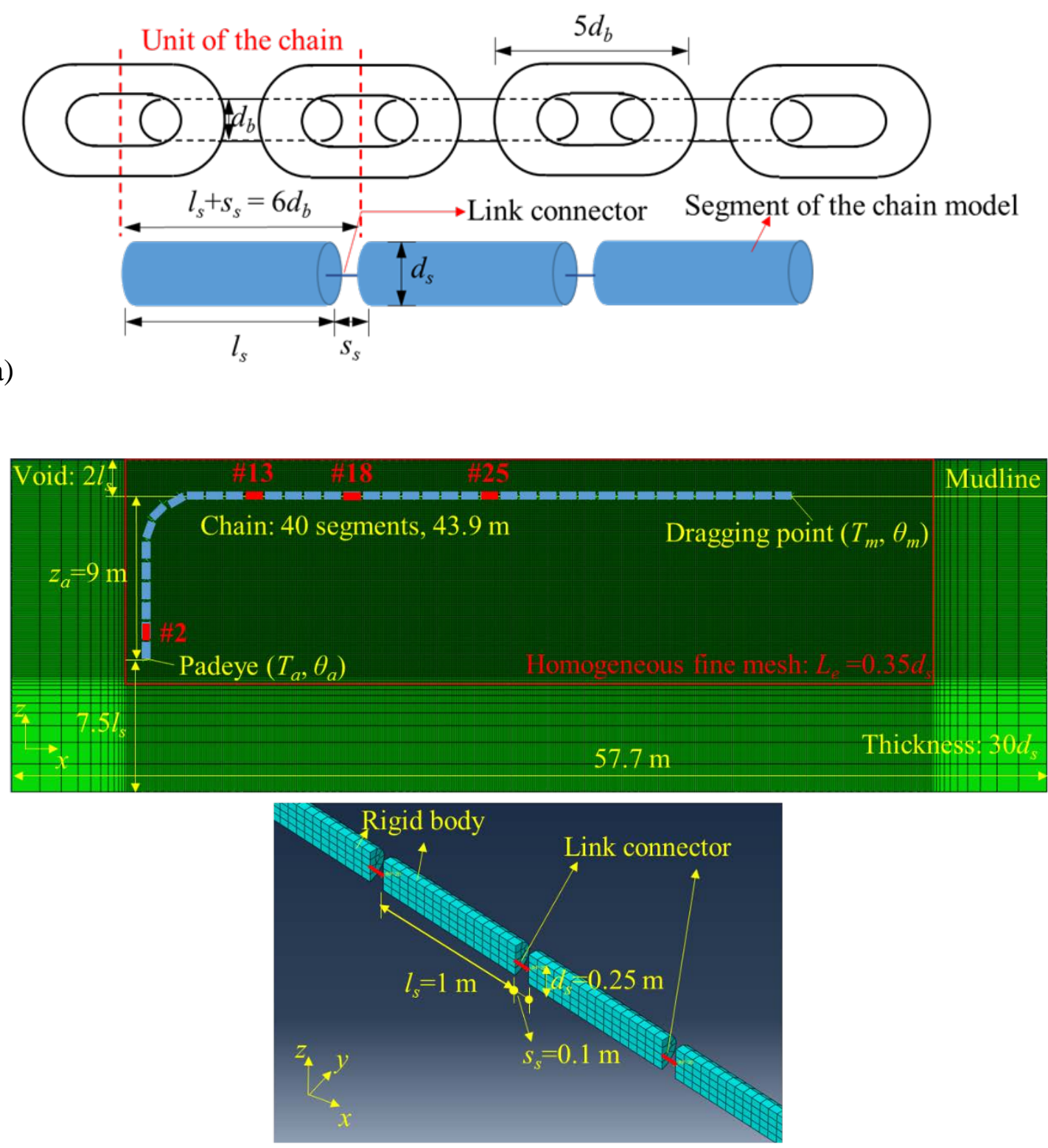

(b)

643 Figure 2. CEL modelling of the embedded chain: (a) Chain model representing $5 d_{b}$ studless chain; (b) FE model setup. 


\section{Sea surface}

Mooring line length in water: $1000 \mathrm{~m}$

Water depth: $475 \mathrm{~m}$

Equilibrium equation of a mooring line: $d T=w_{\text {mooring }} \sin \theta d s$ $\operatorname{Td} \theta=w_{\text {mooring }} \cos \theta d s$
Section \#3: Connection chain $75 \mathrm{~m}$, $w_{\text {mooring }}=1.96 \mathrm{kN} / \mathrm{m}$

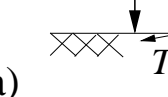
Section \#1: Ground chain $240 \mathrm{~m}, w_{\text {mooring }}=1.37 \mathrm{kN} / \mathrm{m}$
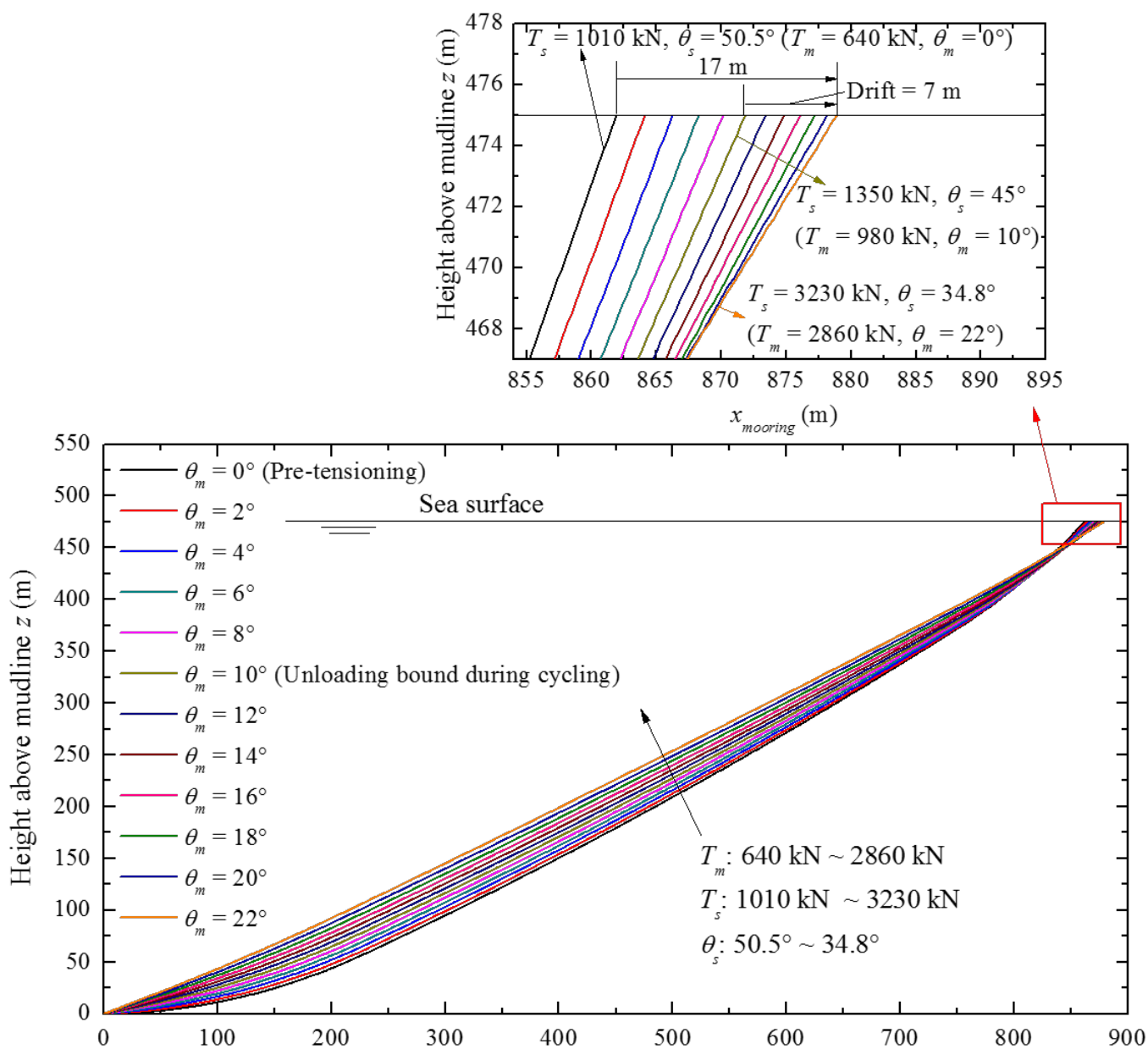
mooring line in the seawater; (b) Configurations of the mooring line and the corresponding boundaries of the tension and angle. 
650

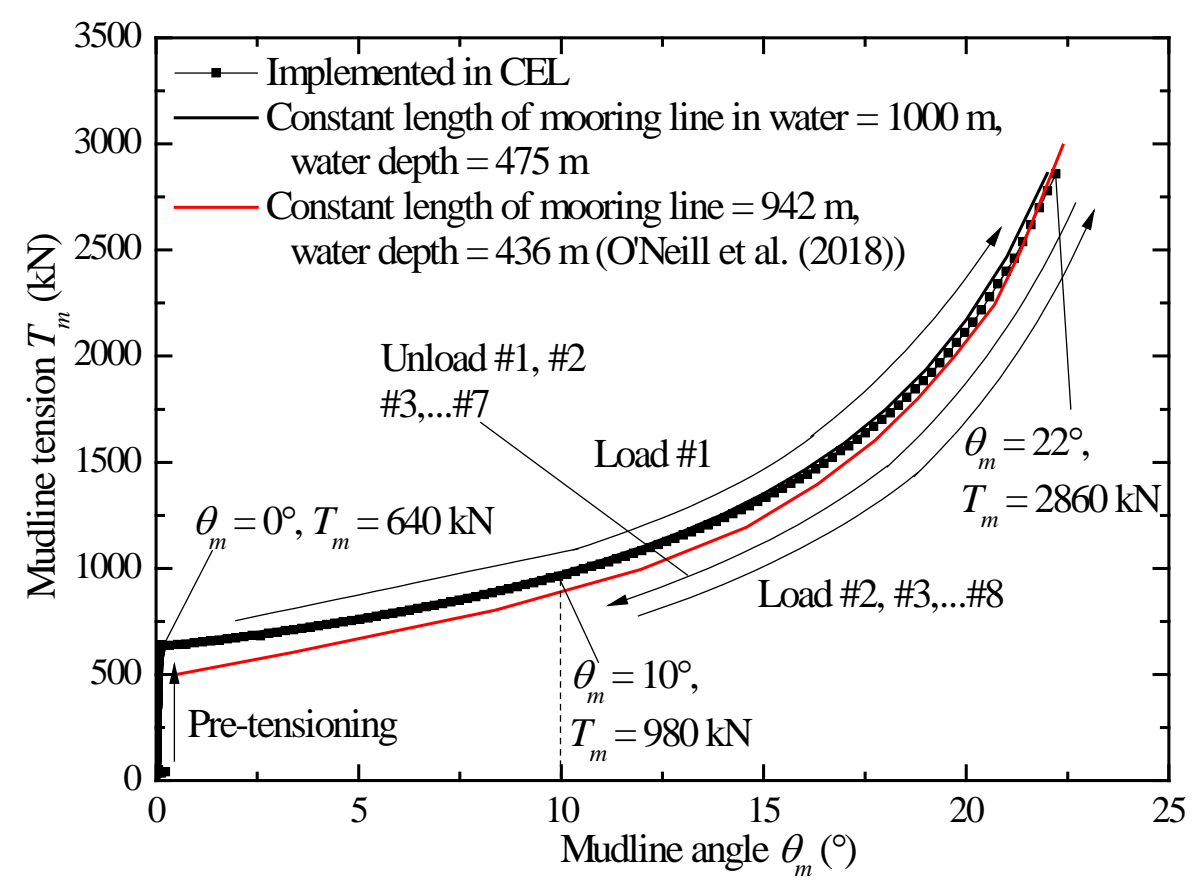

652

Figure 4. Load input from the mooring line in the seawater. 


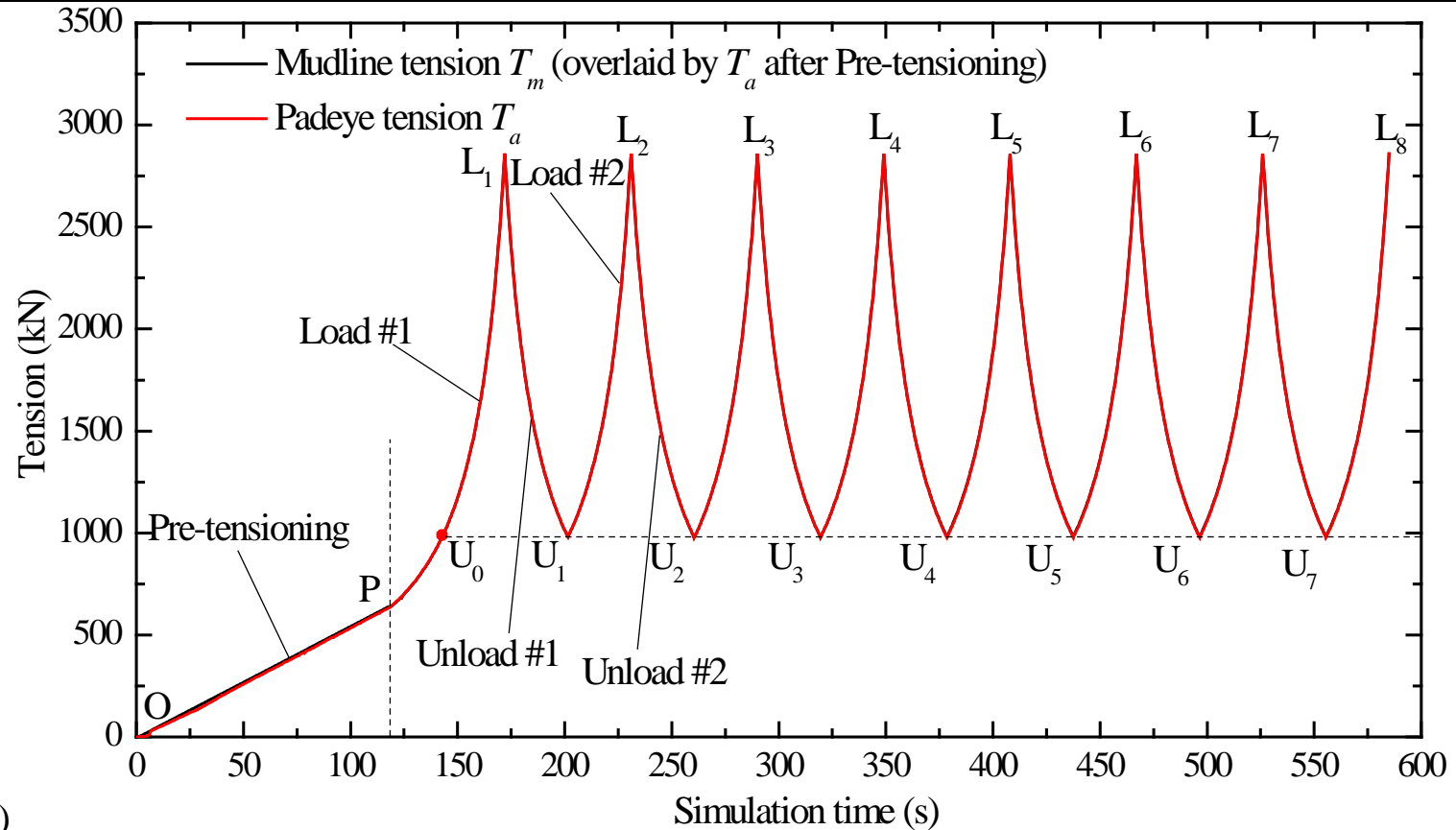

653

(a)

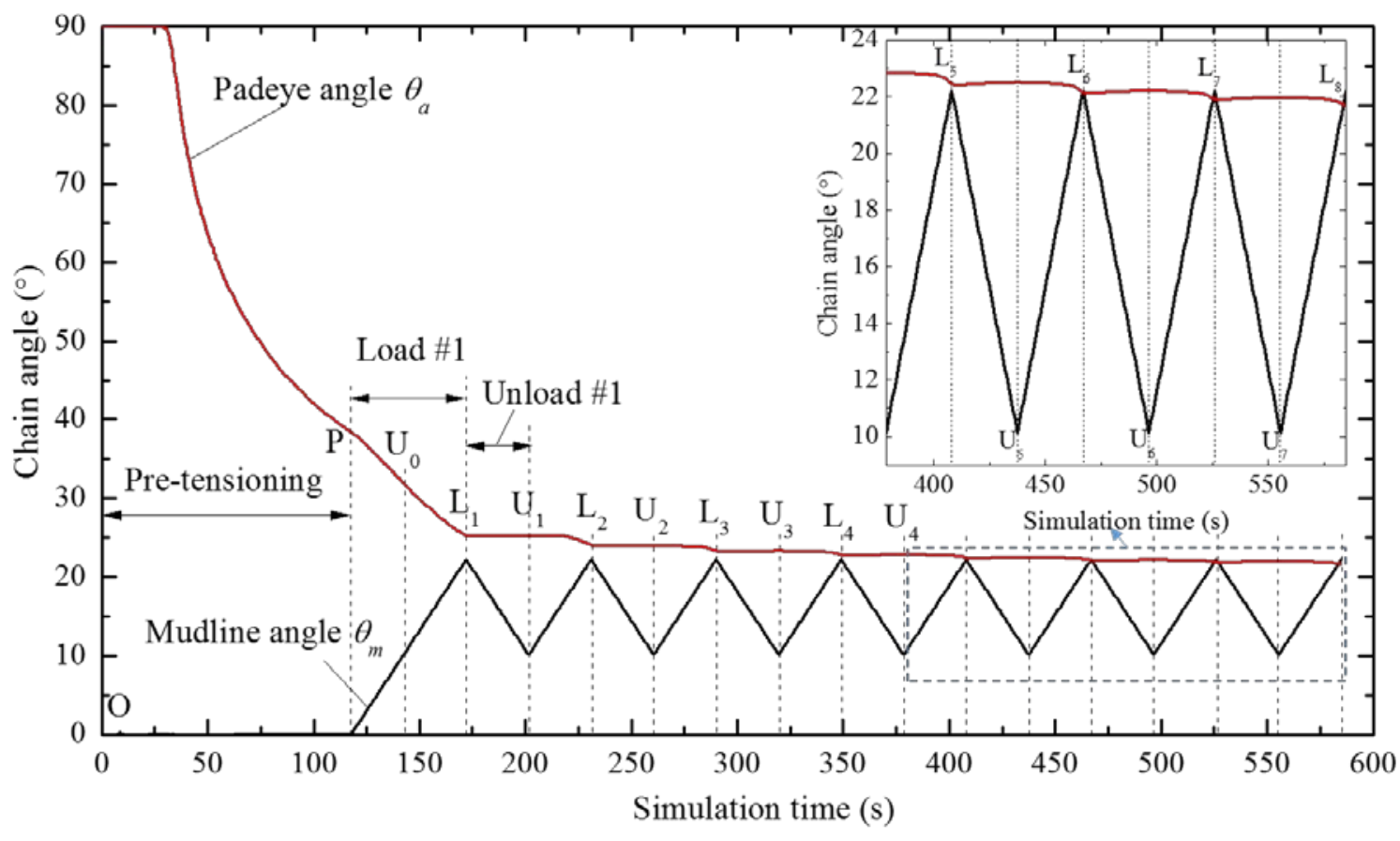

655 Figure 5. Tensions and angles of the chain during the complete simulation: (a) Mudline and padeye tension; (b) Mudline and padeye angles. 


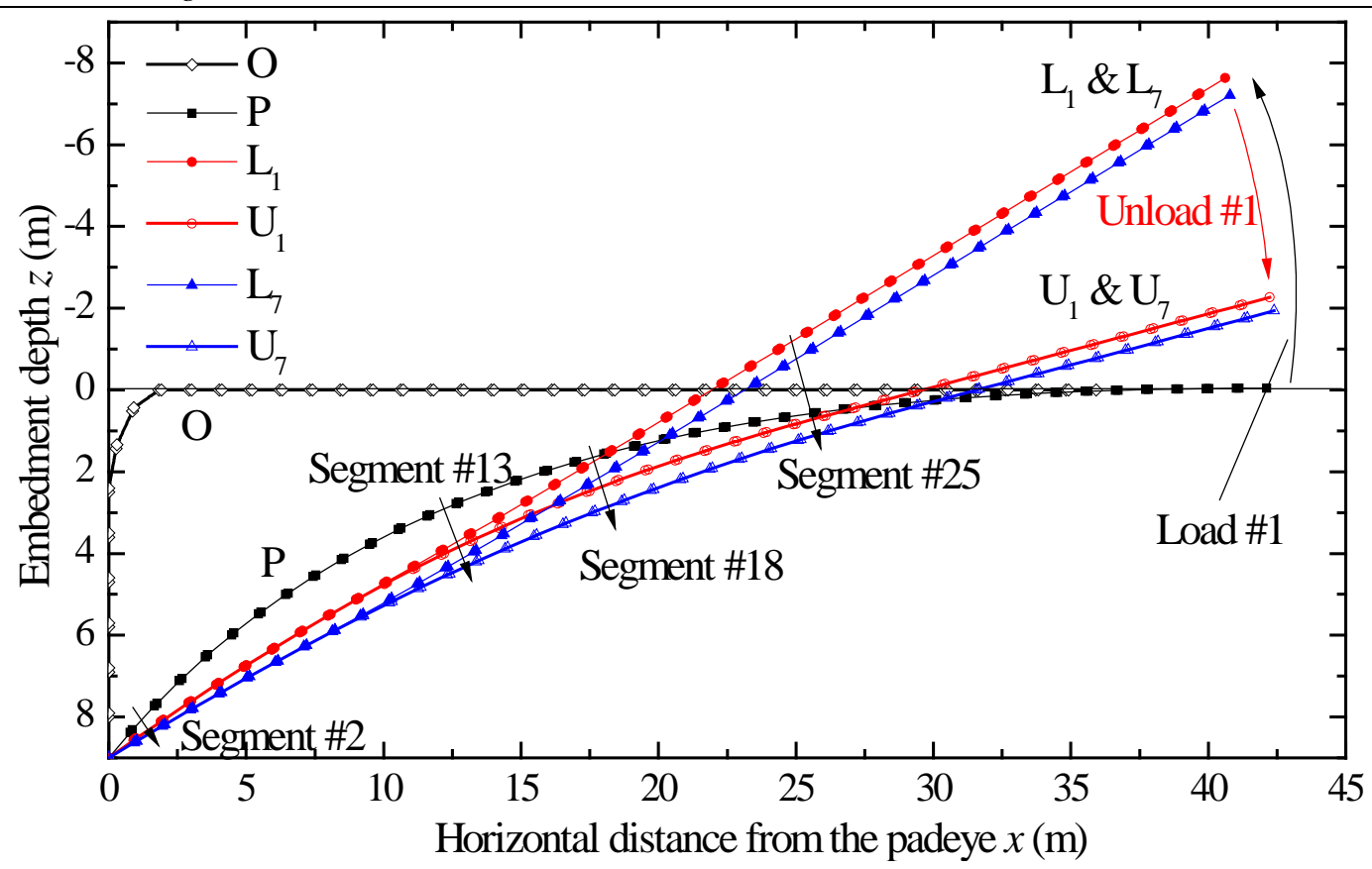

658 Figure 6. Comparison of the configurations of the embedded chain at selected instants during cyclic loading. 


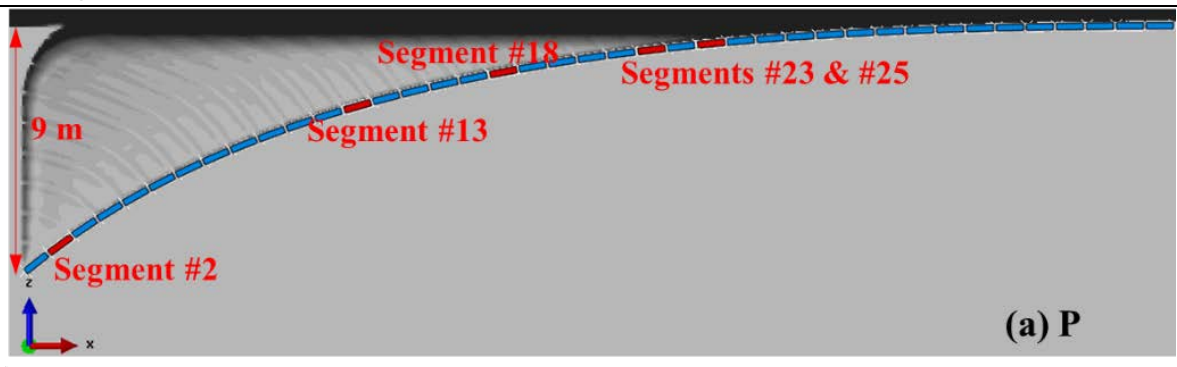

661
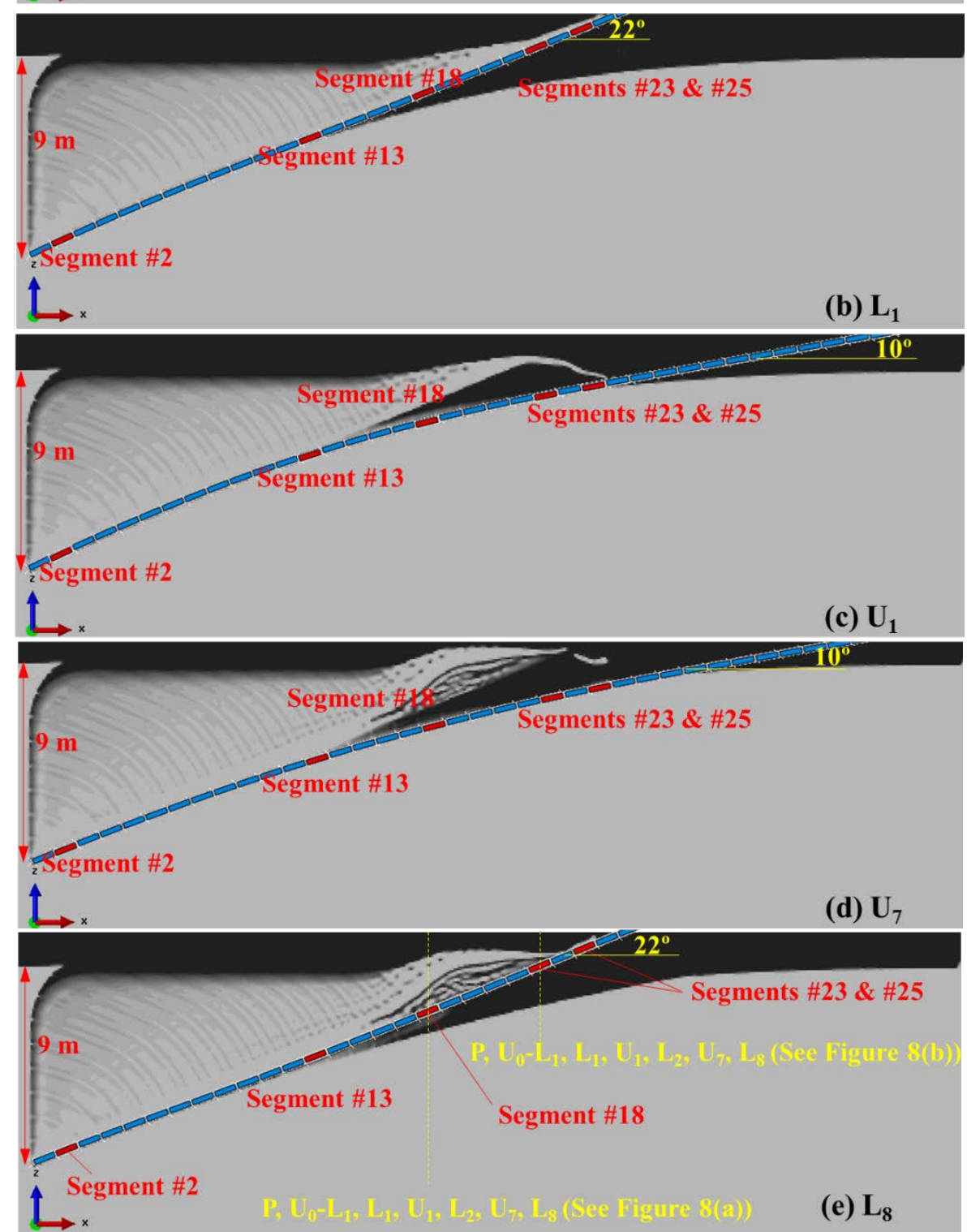

666

Figure 7. Volume fraction of soil at selected instants during cyclic loading. 

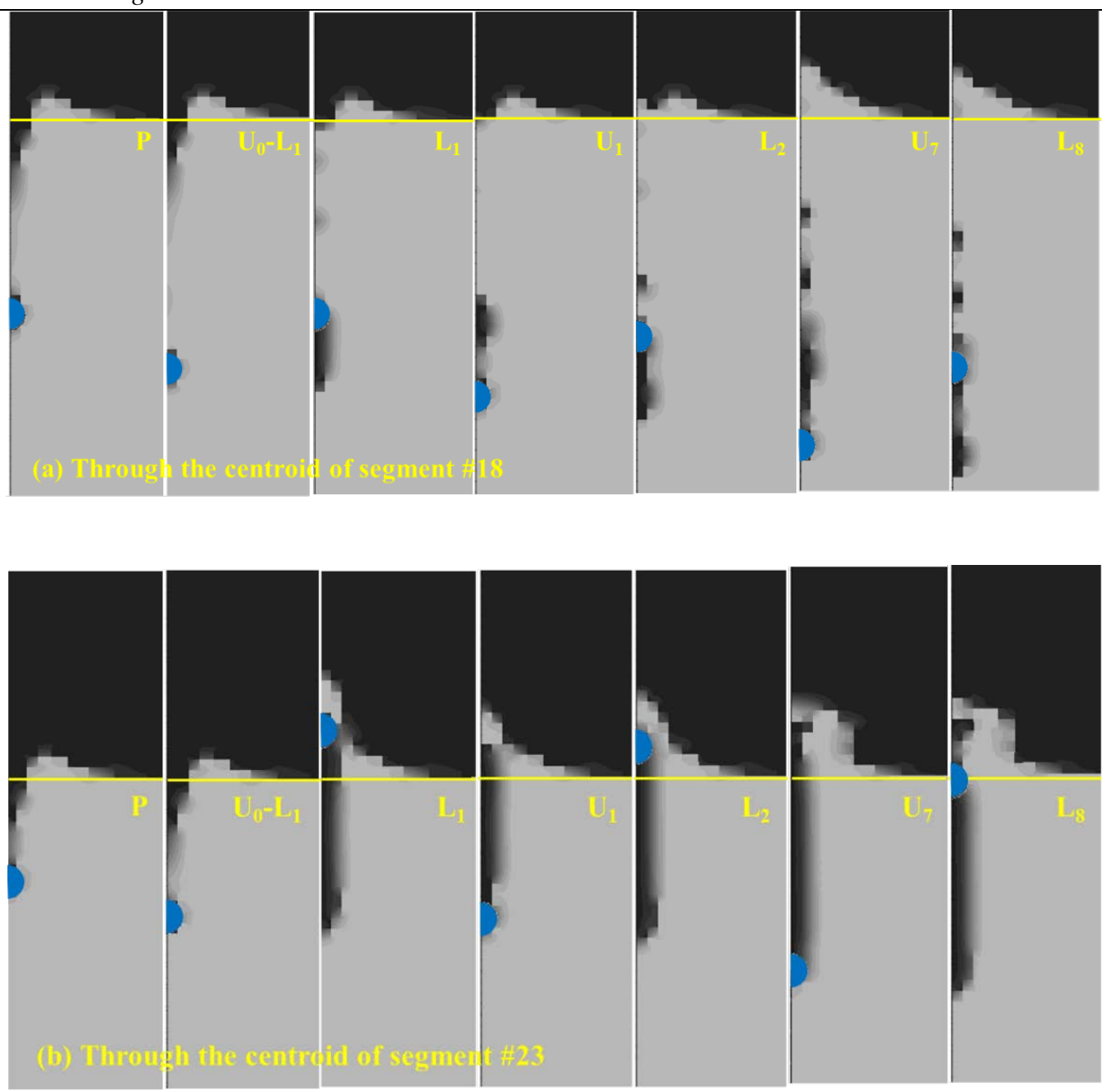

Figure 8. Cross-sectional views of the volume fraction of soil at selected instants during cyclic loading. 

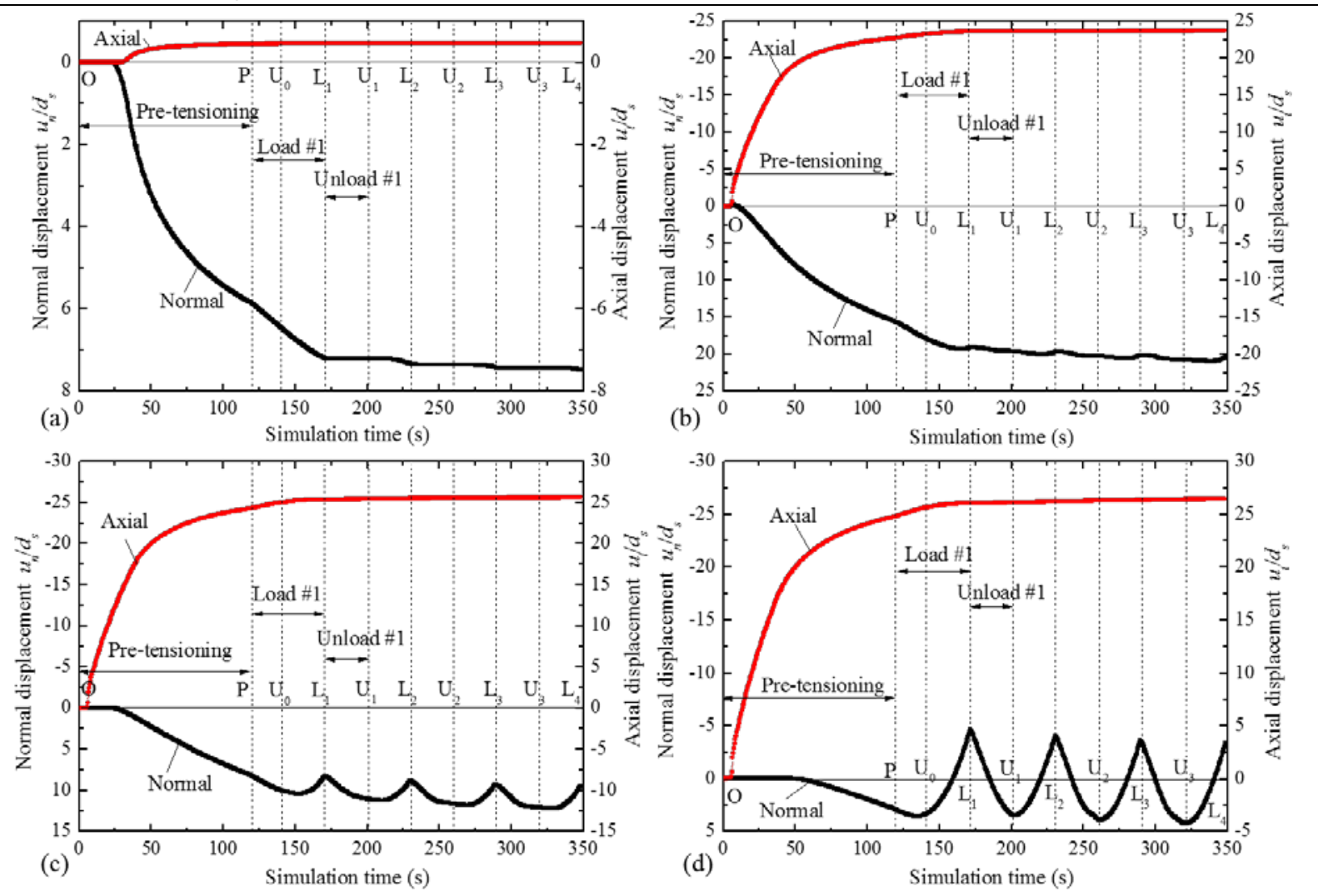

672 Figure 9. Accumulated displacement of selected chain segments (from pre-tensioning to Load \#4): (a) Segment \#2; (b) Segment \#13; (c) Segment \#18; (d) Segment \#25. 

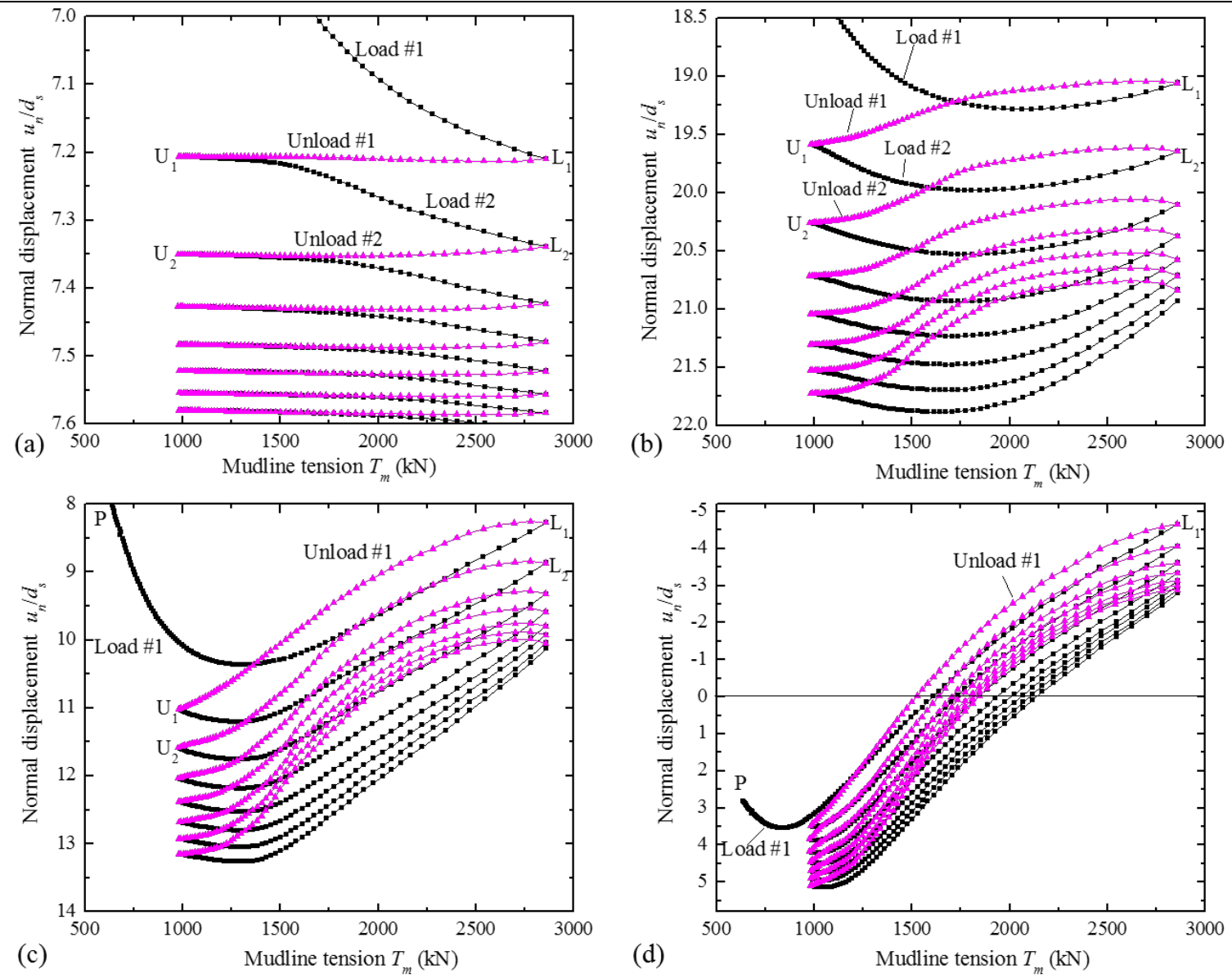

Figure 10. Variation of normal displacement during cyclic loading for selected chain

(d) Segment \#25. 

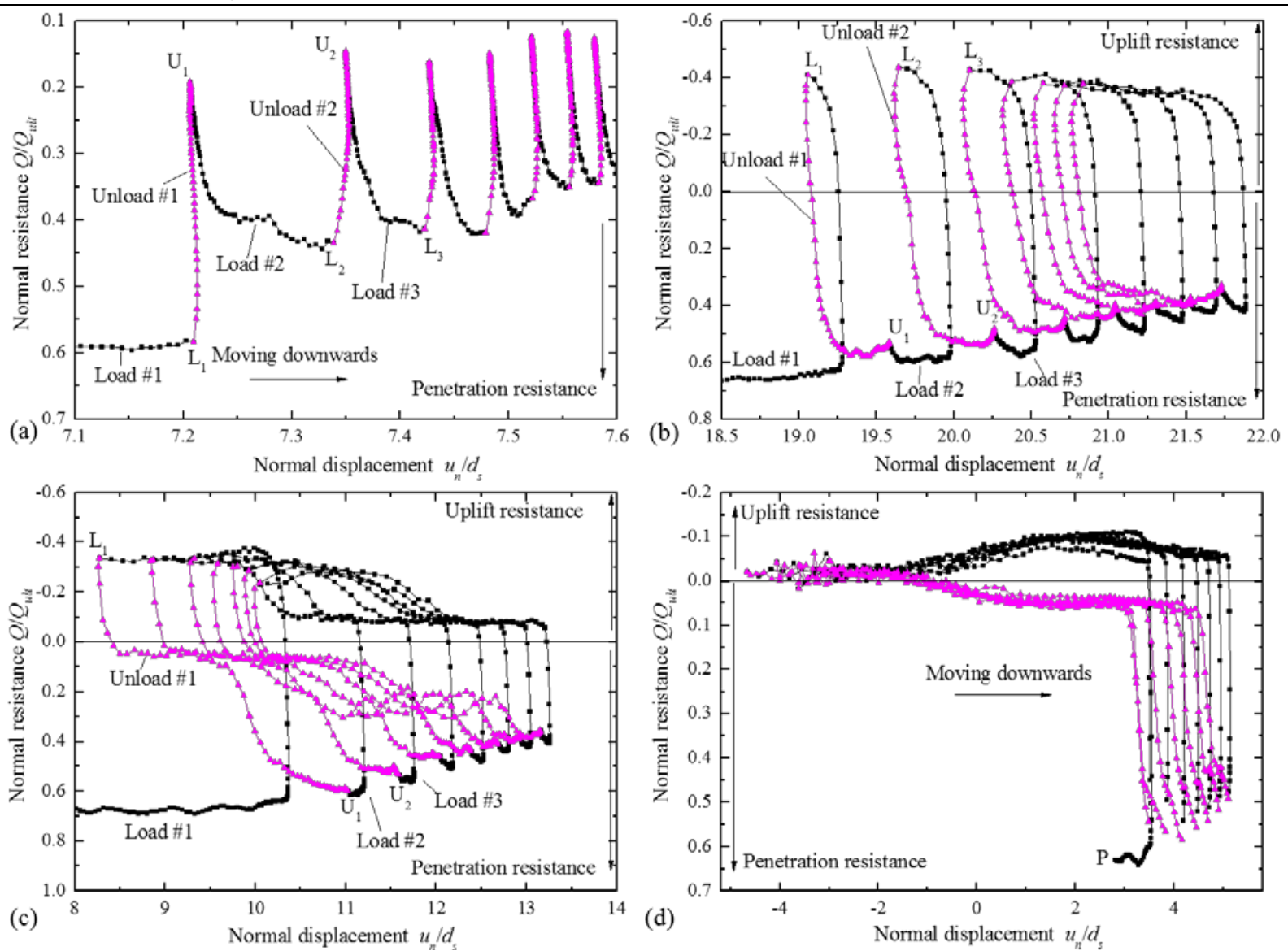

678 Figure 11. Normal resistance-displacement response for selected chain segments: (a) Segment \#2; (b) Segment \#13; (c) Segment \#18; (d) Segment \#25. 

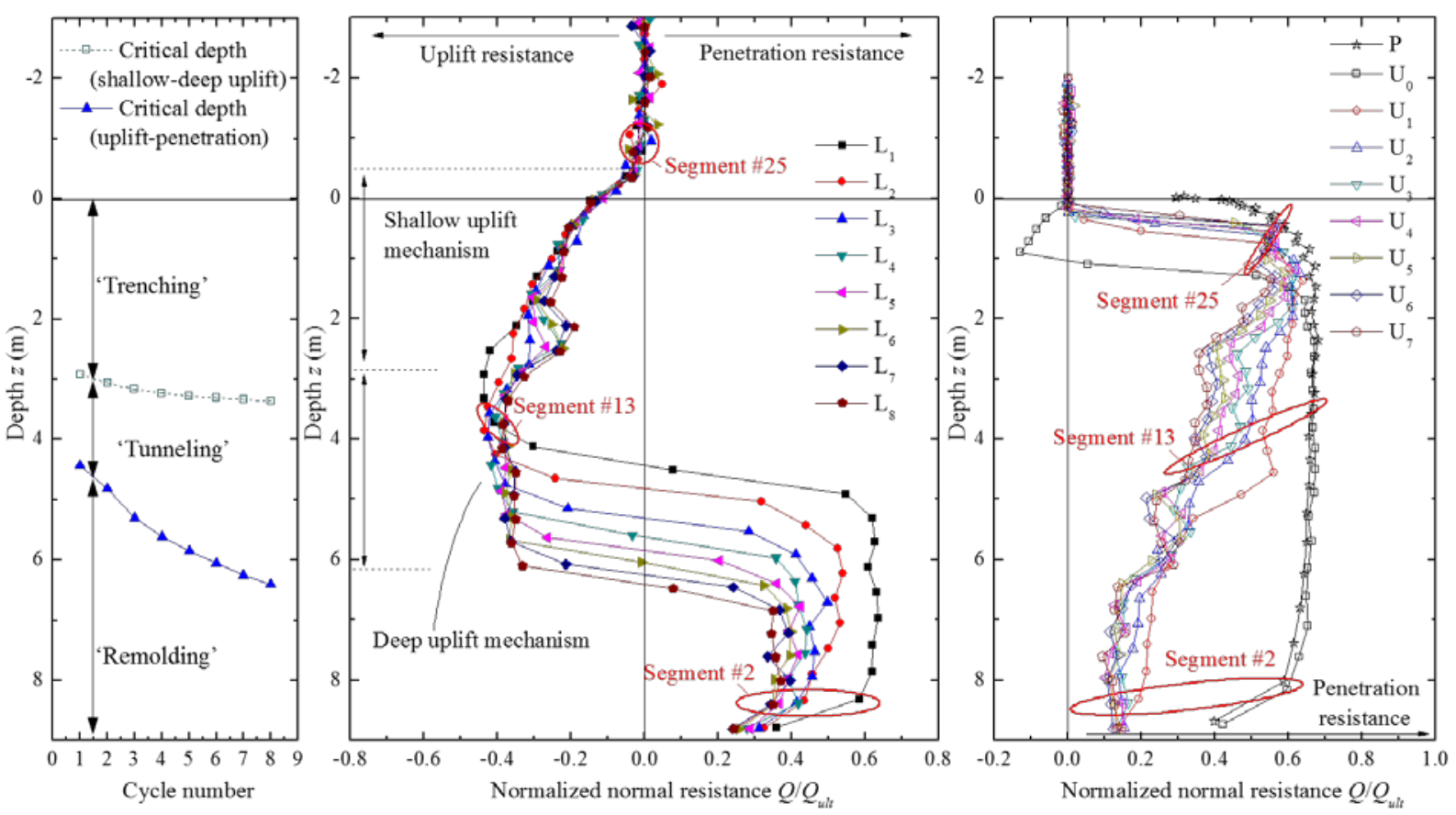

682 Figure 12. Evolution of the depth profile of normal soil resistance to the chain at selected instants during cyclic loading. 
684

685
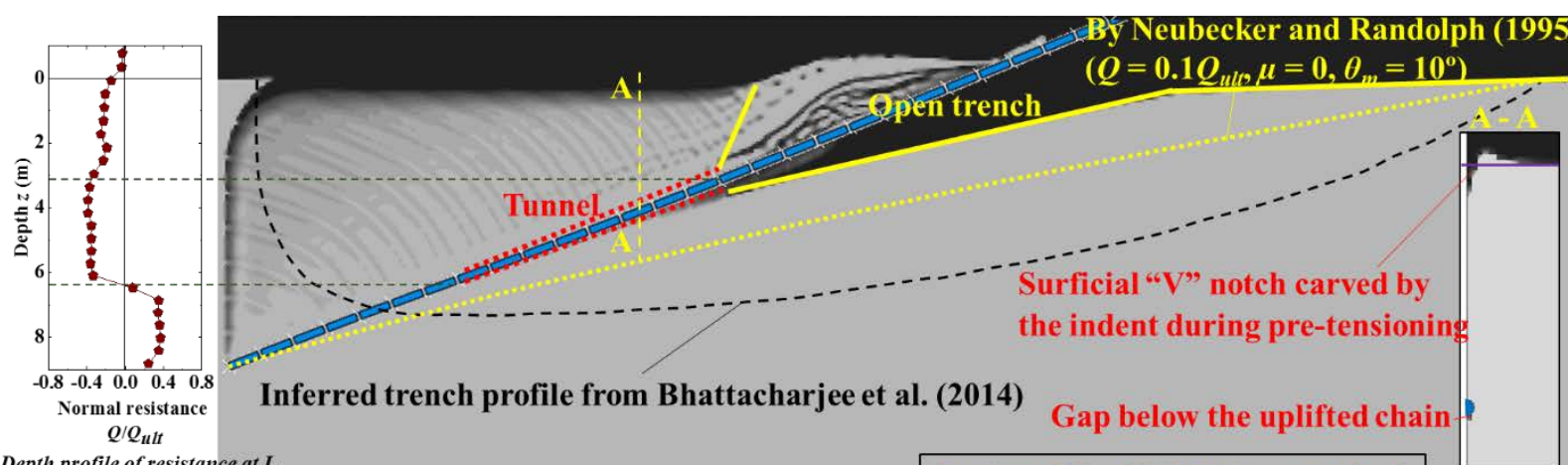

Depth profile of resistance at $L_{s}$

(a)

Tension: $980 \sim 2860 \mathrm{kN}, \mathrm{L}_{8}, s_{u 0}=1.2 \mathrm{z}+2$

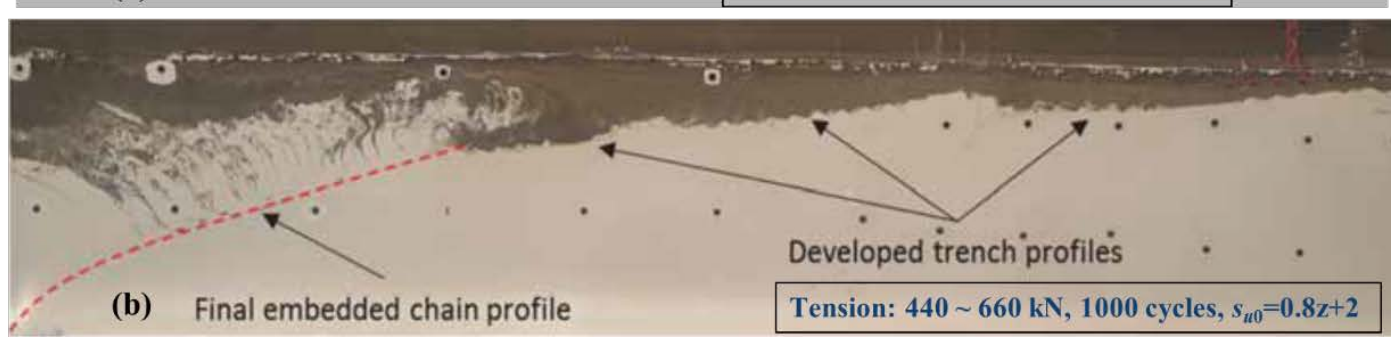

687

Figure 13. Implications about the development of trenching within the scope of current

688 model: (a) CEL modelling; (b) Centrifuge model test. 